\title{
Stability Analysis of a Quarantined Epidemic Model with Latent and Breaking-Out over the Internet
}

\author{
Munna Kumar ${ }^{\mathrm{a}}$, Bimal Kumar Mishra ${ }^{\mathrm{b}}$ and T. C. Panda ${ }^{\mathrm{c}}$ \\ ${ }^{a}$ Research Scholar,Department of Mathematics, Utkal University, \\ Bhubaneswar \\ Orissa,India - 751004 \\ ${ }^{b}$ Department of Applied Mathematics, Birla Institute of Technology \\ Mesra, Ranchi, India - 835215 \\ ${ }^{c}$ Former Principal, Orissa Engineering College, Bhubaneswar \\ Orissa, India - 752050 \\ indian68mk@gmail.com ${ }^{a}$,drbimalmishra@gmail.com ${ }^{b}$, \\ tc_panda@yahoo.com ${ }^{c}$
}

\begin{abstract}
In this paper, a new epidemic model, known as the SLBQRS model, is proposed in a computer network with Latent and Breaking-out periods of a virus in its life cycle. Furthermore, an infected computer will be referred to as latent or breaking-out depending on whether all viruses staying in it are in their respective latent periods or at least one virus staying in it is in its breaking-out period. In the real world there exists no infected computer at all that has no infectivity. A breaking-out computer can get treated with a higher probability, because it usually suffers from a marked performance degradation or even breaks down, which can be perceived evidently by the user. The quarantine is a method of isolating the most infected nodes from the network and reduce the spreading virus till they get recovered. The qualitative properties of this model are investigated. The result shows that the dynamic behavior of the model is determined by a threshold $R_{0}$. Specially, virus-free equilibrium is globally asymptotically stable if $R_{0} \leq 1$. Next, the sensitivity analysis of $R_{0}$ to six system parameters is also analyzed. On this basis, a collection of strategies are advised for eradicating viruses spreading across the Internet effectively. Threshold $R_{0}$, equilibrium and their stability are discussed in terms of the Jacobian of the system. Numerical methods and MATLAB are employed to solve and simulate the system of equations developed and analysis of the model gives remarkable exposure.
\end{abstract}

Keywords: Epidemic model; Computer virus; Latent; Breaking-out; Quarantine; Equilibrium; Basic reproduction Number; Global stability; Infected computer network

\section{Introduction}

With the rapid development of computer technologies and network applications, the Internet has become a powerful mechanism for propagating computer virus. Because of this, computers connected to the Internet become much vulnerable to digital threats. It is quite urgent to understand how computer viruses spread over the Internet and to propose effective measures to cope with this issue. To achieve this goal, and in view of the fact that the spread of virus among computers resembles that of biological virus among a population, it is suitable to establish dynamical models describing the propagation of computer viruses across the Internet by appropriately modifying epidemic models [1]. 
Some classic epidemic models were established for computer virus propagation, such as the SIRS model [2-6], the SEIR model [7], the SEIRS model [8], the SEIQV model [9] and the SEIQRS model [10], which share a common assumption that an infected computer in which the virus resides is in latency cannot infect other computers. Recently, Yang et al. [11-14] proposed a new model, the SLBS model, by taking into account the fact that a computer immediately possesses infection ability once it is infected. This model, however, does not consider that those recovered computers can gain temporary immunity.

Very recently, Yang et al. [15] proposed a new model, the SLBRS model, which incorporates an additional recovery compartment having temporary immunity from which virus is removed after its outbreak. This model also, however, does not consider the quarantine computers that can play an important role in the recovery of the nodes.

Extending the SLBRS model of Yang et al. [15], new compartment quarantine has been introduced and effect of quarantine has been analyzed in this paper.

One way to control the spread of virus for the nodes which are highly infected is to be kept in isolation for some time. Isolation may have been the first infection control method in biological diseases. The word quarantine means to say about the forced isolation or stoppage of interaction with others. Same concept has used in computer world. This may help us to reduce the transmission of infection to susceptible nodes. The infected files are quarantined to reduce the further transmission of malicious objects in computer network.

In this paper, we propose a new computer virus model, the SLBQRS model, which incorporates an additional quarantine compartment. In SLBQRS model, all the computers connected to the Internet are partitioned into five compartments: uninfected computers having no immunity (S computers), infected computers that are latent (L computers), infected computers that are breaking out (B computers), infected computers that are quarantined ( $\mathrm{Q}$ computers) and uninfected computers having temporary immunity (R computers) from which virus is removed after its outbreak. Because the incidence rate of SLBQRS model is $f(S ; L, B)$, not conventional $f(S, I)[7,10]$, it is a challenge to research this new model.

It is proved that the dynamical behavior of the SLBQRS model is fully determined by a threshold $R_{0}$ : the virus-free equilibrium is globally asymptotically stable if $R_{0} \leq 1$. The theoretical results obtained imply some practical means of eradicating computer viruses distributed over the Internet.

The subsequent materials are organized in this pattern: Section 1 deals with introduction of the paper. Section 2 elucidates principle of the model. Section 3 formulates the SLBQRS model. Section 4 gives its basic reproduction number $R_{0}$. Section 5 gives its solutions and stability and examine the global stability of the virusfree equilibrium. Section 6 presents Numerical Methods and simulation. Section 7 Poses a set of effective strategies for controlling the spread of computer virus across the Internet by analyzing the dependence of $R_{0}$ on the system parameters. Finally, Section 8 makes a brief summary of this work.

\section{Nomenclature:}

- S: Uninfected computers having no immunity.

- L: Infected computers that are latent.

- B: Infected computers that are breaking out.

- Q: Infected computers that are quarantined.

- R: Uninfected computers having temporary immunity.

- $\mu$ : Rate at which External computers are connected to the Internet.

- $\mu$ : Rate at which internal computers are disconnected from the Internet.

- $\lambda$ : Rate of crashing of the nodes due to the attack of viruses. 
- $\quad \beta$ : Infectious contact rate, i.e., the rate of infection per susceptible and per infective.

- $\alpha$ : Nonnegative constant rate at which Latent computers break out.

- $\eta$ : Nonnegative constant rate at which Breaking-out computers are cured.

- $\gamma$ : Nonnegative constant rate at which Breaking-out computers are quarantined.

- $\delta$ : Nonnegative constant rate at which Quarantined computers are cured.

- $\varepsilon$ :Nonnegative constant rate at which Recovered computers become susceptibly virus-free again.

- $R_{0}$ : Basic reproduction number.

\section{Principle of the Model}

\subsection{Basic Terminologies}

For convenience, let us introduce the following terminologies:

Internal or external computer: A computer is referred to as internal or external depending on whether it is connected to the Internet or not.

Infected or uninfected: A computer is referred to as infected or uninfected depending on whether there is a virus staying in it or not.

Host computer: A computer is referred to as the host computer of a virus if the virus has entered it and is staying in it.

Life cycle of a virus: The interval from the time it enters its host computer to the time it is eradicated.

Lifetime of a virus: The length of its life cycle. The lifetime of a virus is not fixed. Rather, it is affected by a multiplicity of factors.

Latent period: The interval from the time the virus enters its host computer to the time exactly before it inflicts damage on the host system.

Breaking-out period: The interval from the time the virus begins to inflict damage to the time it is wiped out.

\subsection{Principle of Computer Viruses}

A clever computer virus would try to stealthily infect as many computers systems as possible before it finally breaks out. As thus, a typical virus would undergo two consecutive phases: a latent period and breaking-out period. In this paper, we will always assume that an infected computer will be referred to as latent or breaking-out depending on whether all viruses staying in it are in their respective latent periods or at least one virus staying in it is in its breaking-out period.

\section{SLBQRS Model}

In this paper, all computers $(\mathrm{N})$ connected to the Internet are partitioned into five compartments: uninfected computers having no immunity ( $\mathrm{S}$ computers), infected computers that are latent (L computers), infected computers that are breaking out (B computers), infected computers that are quarantined ( $\mathrm{Q}$ computers), and uninfected computers having temporary immunity (R computers). 
Let $\mathrm{S}(\mathrm{t}), \mathrm{L}(\mathrm{t}), \mathrm{B}(\mathrm{t}), \mathrm{Q}(\mathrm{t})$ and $\mathrm{R}(\mathrm{t})$ denote their corresponding percentages of all computers $(\mathrm{N})$ at time $\mathrm{t}$, respectively.

That is,

$$
\mathrm{S}(\mathrm{t})+\mathrm{L}(\mathrm{t})+\mathrm{B}(\mathrm{t})+\mathrm{Q}(\mathrm{t})+\mathrm{R}(\mathrm{t})=\mathrm{N}(\mathrm{t})
$$

In the SLBQRS model for infections that do not confer immunity, when connected to the internet susceptible nodes in S computers first goes through a latent period in L computers after infection, thereafter some infected nodes are breaking out and stay in $B$ computers while they are infectious and then move to the removed computers $R$ upon temporary recovery after the run of anti-malicious software. Other infected nodes are transferred into the quarantine computers $Q$ while they are infectious and then move to the $R$ computers. Since in the cyber world the acquired immunity is not permanent, the recovered nodes return back to the susceptible computers $\mathrm{S}$.

\section{The Following Assumptions Are Made To Characterize The Model:}

- All newly connected computers are all virus free.

- External computers are connected to the Internet at positive constant rate $\mu$. Also, internal computers are disconnected from the Internet at the same rate $\mu$.

- Each virus-free computer gets contact with an infected computer at a bilinear incidence rate $\beta S(L+B)$, where $\beta$ is positive constant.

- Latent computers break out at nonnegative constant rate $\alpha$.

- Breaking-out computers are quarantined at nonnegative constant rate $\gamma$.

- Breaking-out computers are cured at nonnegative constant rate $\eta$.

- Quarantined computers are cured at nonnegative constant rate $\delta$.

- Recovered computers become susceptibly virus-free again at nonnegative constant rate $\varepsilon$

Our assumptions on the transmission of viruses in computer nodes are depicted in figure1.

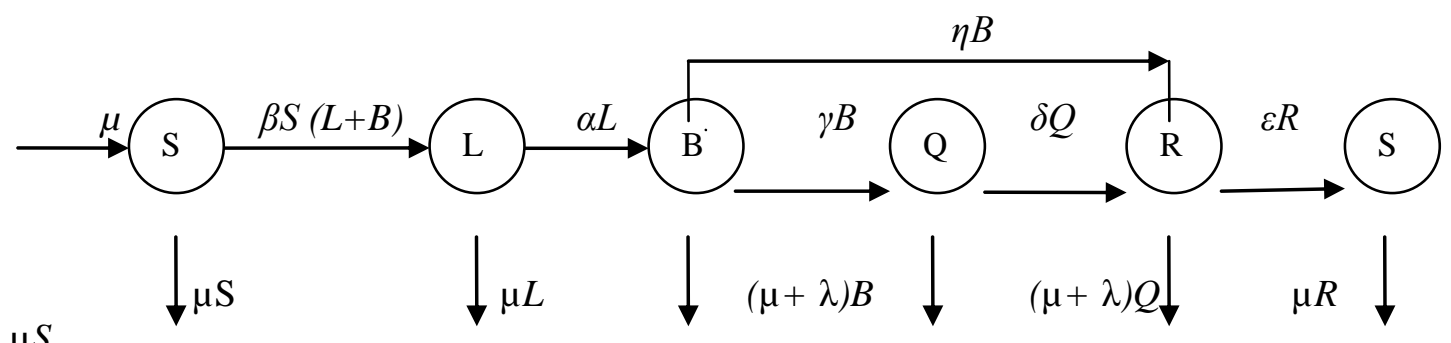

Figure 1. State Transition Diagram for the Flow of Viruses In The SLBQRS Model

Therefore, the transmission between model classes can be expressed by the following system of differential equations:

$$
\begin{gathered}
\frac{d S}{d t}=\mu-\beta S(L+B)+\varepsilon R-\mu S \\
\frac{d L}{d t}=\beta S(L+B)-(\mu+\alpha) L
\end{gathered}
$$




$$
\begin{gathered}
\frac{d B}{d t}=\alpha L-(\mu+\gamma+\eta+\lambda) B \\
\frac{d Q}{d t}=\gamma B-(\mu+\lambda+\delta) Q \\
\frac{d R}{d t}=\delta Q-(\mu+\varepsilon) R+\eta B
\end{gathered}
$$

\section{Basic Reproduction Number $\left(R_{0}\right)$}

As one of the most useful threshold parameters mathematically characterizing the spread of

virus, the basic reproduction number, $R_{0}$, is defined as the expected number of secondary cases produced by a single (typical) infection in a population of susceptible computers.

Since the model has three infected classes (L, B, Q), so, to get $R_{0}$, we take only three equations from the system (2) corresponding to these classes. That is,

$$
\begin{gathered}
\frac{d L}{d t}=\beta S(L+B)-(\mu+\alpha) L \\
\frac{d B}{d t}=\alpha L-(\mu+\gamma+\eta+\lambda) B \\
\frac{d Q}{d t}=\gamma B-(\mu+\lambda+\delta) Q
\end{gathered}
$$

We now linearize these equations, we get, $\left[\begin{array}{c}\frac{d L}{d t} \\ \frac{d B}{d t} \\ \frac{d Q}{d t}\end{array}\right]=(\mathrm{F}-\mathrm{V})\left[\begin{array}{c}L \\ B \\ Q\end{array}\right]$, where, $\mathrm{F}, \mathrm{a}$ matrix of rates of infection and $\mathrm{V}$, a matrix of rates of transmission, are defined by, $\mathrm{F}$

$$
=\left[\begin{array}{ccc}
\beta & \beta & 0 \\
0 & 0 & 0 \\
0 & 0 & 0
\end{array}\right] \text { and } \quad \mathrm{V}=\left[\begin{array}{ccc}
\mu+\alpha & 0 & 0 \\
-\alpha & \mu+\gamma+\eta+\lambda & 0 \\
0 & -\gamma & \mu+\lambda+\delta
\end{array}\right] .
$$

Then the basic reproductive number $R_{0}$ is defined as the dominant eigen value of $\mathrm{F}$ $\mathrm{V}^{-1}$. That

$$
\text { Is, } \quad R_{0}=\frac{\beta(\mu+\alpha+\gamma+\eta+\lambda)}{(\mu+\alpha)(\mu+\gamma+\eta+\lambda)}
$$




\section{Solutions and Stability}

The system (2) is defined on the closed, positive invariant set $\mathrm{D}=\{(\mathrm{S}, \mathrm{L}, \mathrm{B}, \mathrm{Q}, \mathrm{R})$; $\mathrm{S}, \mathrm{L}, \mathrm{B}, \mathrm{Q}, \mathrm{R} \geq 0: S+L+B+Q+R=N\}$ which has two possible equilibriums, first, the virus-free equilibrium, $E_{0}=\left(S_{0}, L_{0}, B_{0}, Q_{0}, R_{0}\right)=(N, 0,0,0,0)$ and second, the endemic(viral) equilibrium

$E^{*}=\left(S^{*}, L^{*}, B^{*}, Q^{*}, R^{*}\right)$ Which is the interior of $\mathrm{D}$ and can be obtained by taking all the equations of system (2) equal to zero. That is,

$$
\begin{gathered}
S^{*}=\frac{(\mu+\alpha)(\mu+\gamma+\eta+\lambda)}{\beta(\mu+\alpha+\gamma+\eta+\lambda)}=\frac{1}{R_{0}}, L^{*}=\frac{(\mu+\gamma+\eta+\lambda)}{\alpha} B^{*}, \\
Q^{*}=\frac{\gamma}{\mu+\lambda+\delta} B^{*}, R^{*}=\frac{\delta \gamma+\eta(\mu+\lambda+\delta)}{(\mu+\varepsilon)(\mu+\lambda+\delta)} B^{*} \text { and } \\
B^{*}=\frac{\left.\mu \alpha(\mu+\lambda+\delta)(\mu+\varepsilon)\left(1-R_{0}\right)\right]}{R_{0} \alpha \gamma \delta \varepsilon+(\mu+\lambda+\delta)\left[R_{0} \alpha \eta \varepsilon-\beta(\mu+\varepsilon)(\mu+\alpha+\gamma+\eta+\lambda)\right]} . \\
E^{*}=\left(S^{*}, L^{*}, B^{*}, Q^{*}, R^{*}\right) \in D^{\prime}=D-\left\{E_{0}\right\} .
\end{gathered}
$$

It is clear that the viral equilibrium exists if and only if $R_{0}>1$.

\subsection{Stability of the Virus-Free Equilibrium State}

Theorem 1: The system (2) is locally asymptotically stable if all its eigen values are negative.

Proof: To get the stability the Jacobian matrix, of the system (2) can be taken as:

$$
J=\left[\begin{array}{ccccc}
-\mu & 0 & 0 & 0 & \varepsilon \\
0 & -(\mu+\alpha) & 0 & 0 & 0 \\
0 & \alpha & -(\mu+\gamma+\eta+\lambda) & 0 & 0 \\
0 & 0 & \gamma & -(\mu+\lambda+\delta) & 0 \\
0 & 0 & \eta & \delta & -(\mu+\varepsilon)
\end{array}\right]
$$

Solving, we get the eigen values, $-\mu,-(\mu+\alpha),-(\mu+\gamma+\eta+\lambda)$, $-(\mu+\lambda+\delta)$,

$-(\mu+\varepsilon)<0$, which all are negative hence the system (2) is locally asymptotically stable at virus-free equilibrium point $\mathrm{E}_{0}$.

Lemma 1: If $R_{0}<1$, the virus free equilibrium point $\mathrm{E}_{0}$ is locally asymptotically stable.

If $R_{0}=1, \mathrm{E}_{0}$ is stable. If $R_{0}>1, \mathrm{E}_{0}$ is unstable.

Let,

$$
\begin{aligned}
& f_{\infty}=\lim _{\mathrm{t} \rightarrow \infty} \inf _{\theta \geq \mathrm{t}} f(\theta) \\
& f^{\infty}=\lim _{\mathrm{t} \rightarrow \infty} \sup _{\theta \geq \mathrm{t}} f(\theta)
\end{aligned}
$$

Lemma 2: Assume that a bounded real valued function $f:[0, \infty] \rightarrow \mathrm{R}$ be twice differentiable with bounded second derivative. Let $\mathrm{k} \rightarrow \infty$ and $f\left(\mathrm{t}_{\mathrm{k}}\right)$ converges to $f^{\infty}$ or $f_{\infty}$ then, 


$$
\lim _{k \rightarrow \infty} f^{\prime}\left(t_{k}\right)=0
$$

Theorem 2: if $R_{0}<1$, then virus free equilibrium $\mathrm{E}_{0}$ is globally asymptotically stable.

Proof: From the system (2) we have,

$$
\frac{d S}{d t} \leq \mu-(\mu+\varepsilon) S
$$

A solution of the equation $\frac{d X}{d t} \leq \mu-(\mu+\varepsilon) X$ is a super solution of $\mathrm{S}(\mathrm{t})$.

Since $\mathrm{X}(\mathrm{t}) \rightarrow \frac{\mu}{(\mu+\varepsilon)}$ as $\mathrm{t} \rightarrow \infty$, then for given $\epsilon_{1}>0$, there exit $\mathrm{t}_{0}$ such that

$\mathrm{S}(\mathrm{t}) \leq \mathrm{X}(\mathrm{t}) \leq \frac{\mu}{(\mu+\varepsilon)}+\epsilon_{1}$, for $\mathrm{t} \geq \mathrm{t}_{0}$

Thus, $S^{\infty} \leq \frac{\mu}{(\mu+\varepsilon)}+\epsilon_{1}$

Let $\in_{1} \rightarrow 0$ then $S^{\infty} \leq \frac{\mu}{(\mu+\varepsilon)}$

Similarly the second equation of the system (2) can be expressed as

$$
\frac{d L}{d t}=\beta(L+B)\left(\frac{\mu}{(\mu+\varepsilon)}\right)-(\mu+\alpha) L
$$

Using this the 3rd and 4th equation of the system (2), we have,

$$
\left[\begin{array}{c}
L^{*} \\
B^{*} \\
Q^{*}
\end{array}\right] \leq P\left[\begin{array}{l}
L \\
B \\
Q
\end{array}\right]
$$

$$
\text { Where, } \quad P=\left[\begin{array}{ccc}
-\left(\mu+\alpha-\frac{\beta \mu}{\mu+\varepsilon}\right) & \frac{\beta \mu}{\mu+\varepsilon} & 0 \\
\alpha & -(\mu+\gamma+\eta+\lambda) & 0 \\
0 & \gamma & -(\mu+\lambda+\delta)
\end{array}\right]
$$

let $\mathrm{M} \in R^{+}$, such that

$\mathrm{M} \geq \max \left\{\left(\mu+\alpha-\frac{\beta \mu}{\mu+\varepsilon}\right),(\mu+\gamma+\eta+\lambda),(\mu+\lambda+\delta)\right\}$.

Thus, $P+\mathrm{MI}_{3 \times 3}$ is a strictly positive matrix where $\mathrm{I}_{3 \times 3}$ is an identity matrix. If $\omega_{1}, \omega_{2}$, $\omega_{3}$ are the Eigen value of $P$, then $\omega_{1}+\mathrm{M}, \omega_{2}+\mathrm{M}, \omega_{3}+\mathrm{M}$ are the Eigen value of $P+\mathrm{MI}_{3 \times 3}$. Thus, from the Perron-Frobenius theorem[16], $\mathrm{P}+\mathrm{MI}_{3 \times 3}$ has a simple positive Eigen value equal to dominant Eigen value and corresponding Eigen vector e $>0$, which implies that, $\omega_{1}, \omega_{2}$ and $\omega_{3}$ are real. If $\omega_{1}+\mathrm{M}$ is the dominant Eigen value of $\mathrm{P}+\mathrm{MI}_{3 \times 3}$, then $\omega_{1}>\omega_{2}$ and $\omega_{1}>\omega_{3}$, and $\mathrm{e}^{p}=\mathrm{e}^{\omega 1}$. Obviously $\omega_{1}, \omega_{2}$ and $\omega_{3}$ are the roots of equation.

$$
\omega^{3}+G \omega^{2}+H \omega+K=0
$$


Where $G=\left(3 \mu+\alpha+\gamma+\eta+2 \lambda+\delta-\frac{\beta \mu}{(\mu+\varepsilon)}\right)$,

$H=\left[\left(\mu+\alpha-\frac{\beta \mu}{(\mu+\varepsilon)}\right)(\mu+\gamma+\eta+\lambda)+\left(\mu+\alpha-\frac{\beta \mu}{(\mu+\varepsilon)}\right)(\mu+\lambda+\delta)+(\mu+\gamma+\eta+\lambda)(\mu+\lambda+\delta)-\frac{\beta \mu \alpha}{(\mu+\varepsilon)}\right]$

and $K=\left[\left(\mu+\alpha-\frac{\beta \mu}{(\mu+\varepsilon)}\right)(\mu+\gamma+\eta+\lambda)(\mu+\lambda+\delta)-\frac{\beta \mu \alpha(\mu+\lambda+\delta)}{(\mu+\varepsilon)}\right]$

Since $\mathrm{R}_{0}<1$ for $\epsilon_{1}>0$, sufficiently small, we have,

$$
\left[\left(\mu+\alpha-\frac{\beta \mu}{(\mu+\varepsilon)}\right)(\mu+\gamma+\eta+\lambda)(\mu+\lambda+\delta)-\frac{\beta \mu \alpha(\mu+\lambda+\delta)}{(\mu+\varepsilon)}\right]>0
$$

Therefore coefficients of the equation (6) are positive.

Thus $\omega_{1}, \omega_{2}$ and $\omega_{3}$ are negative. So from equation (5) for $t \geq t_{0}$

$$
\frac{d}{d t}(e[L(t), B(t), Q(t)]) \leq \omega_{1} \cdot e[L(t), B(t), Q(t)]
$$

Integrating the above inequality, we get,

$0 \leq e \cdot[L(t), B(t), Q(t)] \leq e \cdot\left[L\left(t_{1}\right), B\left(t_{1}\right), Q\left(t_{1}\right)\right] e^{\omega_{1}\left(t-t_{1}\right)}$, for $\mathrm{t} \geq \mathrm{t}_{1} \geq \mathrm{t}_{0}$.

Since $\omega_{1}<0$, e. $\left[\mathrm{L}\left(\mathrm{t}_{1}\right), \mathrm{B}\left(\mathrm{t}_{1}\right), \mathrm{Q}\left(\mathrm{t}_{1}\right)\right] \rightarrow 0$ as $\mathrm{t} \rightarrow \infty$

Using e $>0$, we have,

$[L(t), B(t), Q(t)] \rightarrow(0,0,0)$ as $\mathrm{t} \rightarrow \infty$

By Lemma 2, we choose a sequence

$\mathrm{t}_{\mathrm{n}} \rightarrow \infty, \mathrm{S}_{\mathrm{n}} \rightarrow \infty(\mathrm{n} \rightarrow \infty)$, such that

$\mathrm{S}\left(\mathrm{S}_{\mathrm{n}}\right) \rightarrow \mathrm{S}^{\infty}, \mathrm{S}\left(\mathrm{t}_{\mathrm{n}}\right) \rightarrow \mathrm{S}_{\infty}, \mathrm{S}\left(\mathrm{S}_{\mathrm{n}}\right) \rightarrow 0, \mathrm{~S}\left(\mathrm{t}_{\mathrm{n}}\right) \rightarrow 0$

Since $\mathrm{L}(\mathrm{t}), \mathrm{B}(\mathrm{t}) \rightarrow \mathrm{Q}(\mathrm{t}) \rightarrow 0$ as $\mathrm{t} \rightarrow \infty$,

Thus from the first equation of system (2), we have,

$\lim _{\mathrm{n} \rightarrow \infty} \mathrm{S}(\mathrm{t})=\frac{\mu}{(\mu+\varepsilon)}$

Hence by incorporating lemma $\mathbf{1}$, the virus-free equilibrium $\mathrm{E}_{0}$ is globally asymptotically stable, if $R_{0}<1$.

\section{Numerical Methods and Simulation}

We present the numerical result using Runge-Kutta method of order 4 and MATLAB are employed to solve and simulate the system (2) and the behavior of the different classes with respect to time are observed which is depicted in figures 2 and we observe that the system is asymptotically stable. The stability of the system is numerically verified for different conditions of the basic reproduction number. For quarantine nodes, it is observed that the system is asymptotically stable when the basic reproduction number $R_{0}$ is less than one (Figure 3). The network is assumed to have initial values: $\mathrm{S}=30 ; \mathrm{L}=3 ; \mathrm{B}=1 ; \mathrm{Q}=0 ; \mathrm{R}=0$. System is asymptotically stable when the basic reproduction number $R_{0}$ is less than one (Figure 3 ).

The network is assumed to have initial values: $\mathrm{S}=30 ; \mathrm{L}=3 ; \mathrm{B}=1 ; \mathrm{Q}=0 ; \mathrm{R}=0$. 


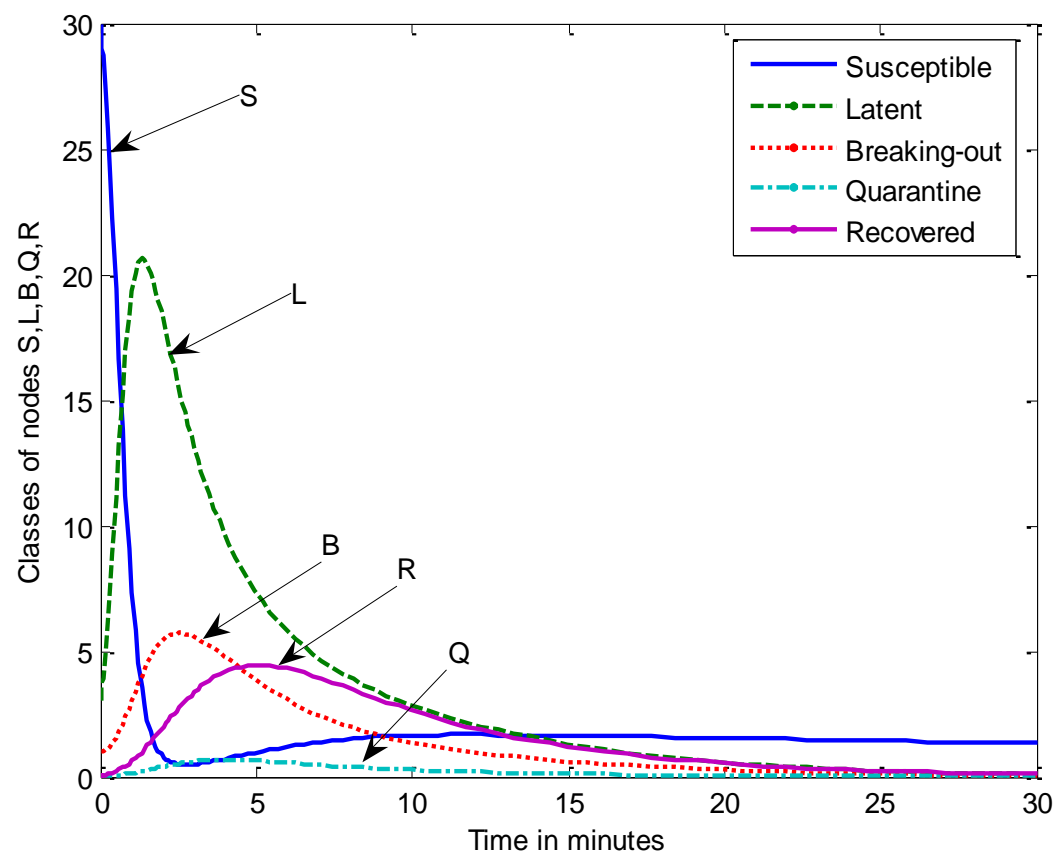

Figure 2. Dynamical Behavior of the System (2) For Different Classes When $\mu=0.1 ; B=0.1 ; A=0.3 ; \Gamma=0.1 ; \Lambda=0.2 ; H=0.4 ; \Delta=0.4 ; E=0.3$;

Fig. 2 shows that all latent computers have infectivity and a breaking-out computer can get treated with a higher probability than latent computer. Infection first increases then become smaller in this breaking-out period.

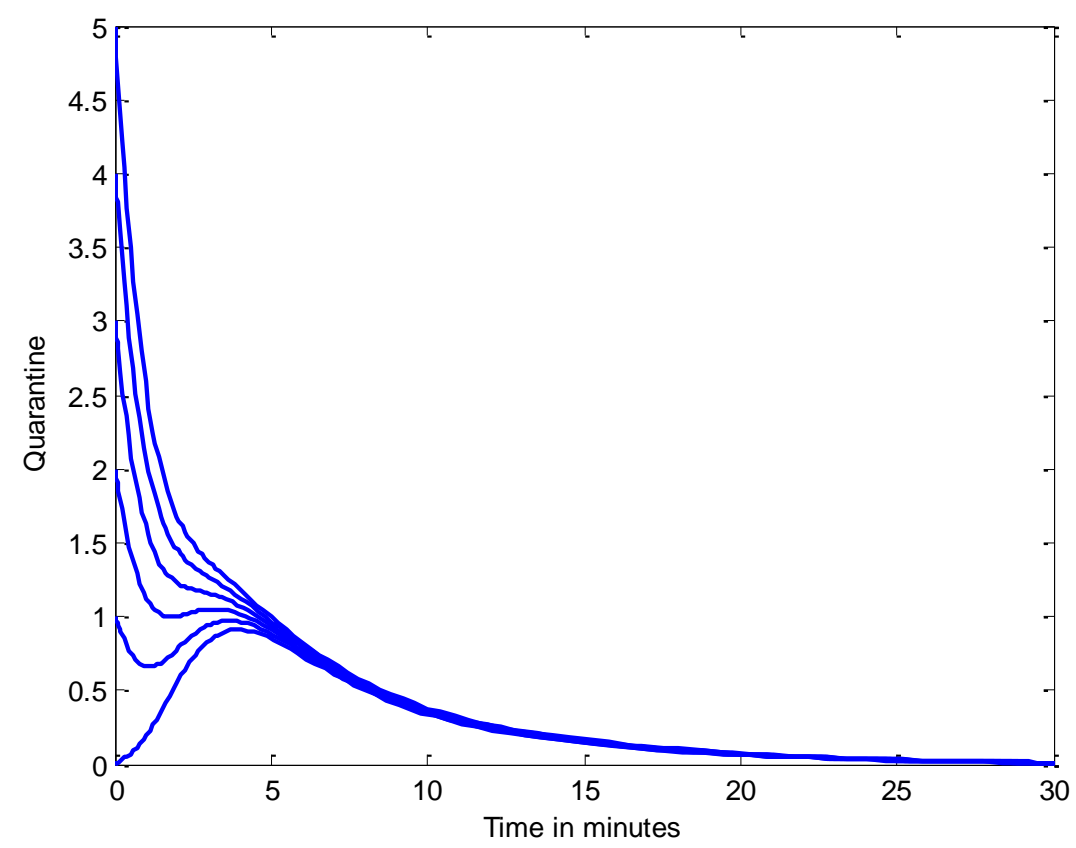

Figure 3. Quarantine Nodes Equilibrium Point with Time When $R_{0}<1$ 


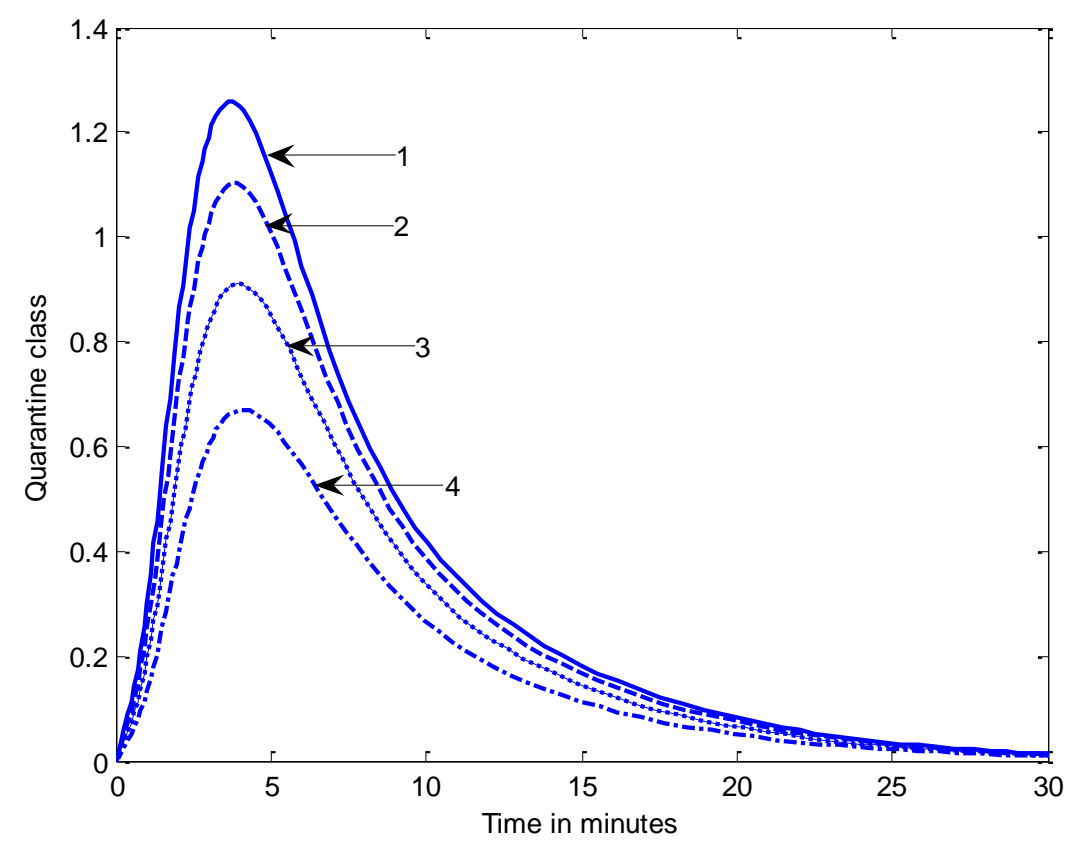

Figure 4. Dynamical Behavior of Quarantine Class With Respect To Time

(1) $\mu=0.1 ; \beta=0.1 ; \alpha=0.3 ; \gamma=0.1 ; \lambda=0.2 ; \eta=0.4 ; \delta=0.4 ; \varepsilon=0.3$;

(2) $\mu=0.1 ; \beta=0.1 ; \alpha=0.3 ; \gamma=0.15 ; \lambda=0.2 ; \eta=0.4 ; \delta=0.45 ; \varepsilon=0.3$;

(3) $\mu=0.1 ; \beta=0.1 ; \alpha=0.3 ; \gamma=0.2 ; \lambda=0.2 ; \eta=0.4 ; \delta=0.5 ; \varepsilon=0.3$;

(4) $\mu=0.1 ; \beta=0.1 ; \alpha=0.3 ; \gamma=0.25 ; \lambda=0.2 ; \eta=0.4 ; \delta=0.55 ; \varepsilon=0.3$

Figure 4 shows a powerful impact of quarantine class. Infection show high infectious behavior in this class but reduces rapidly and get fast recovery to end the susceptibility of spread of infection to benign nodes.

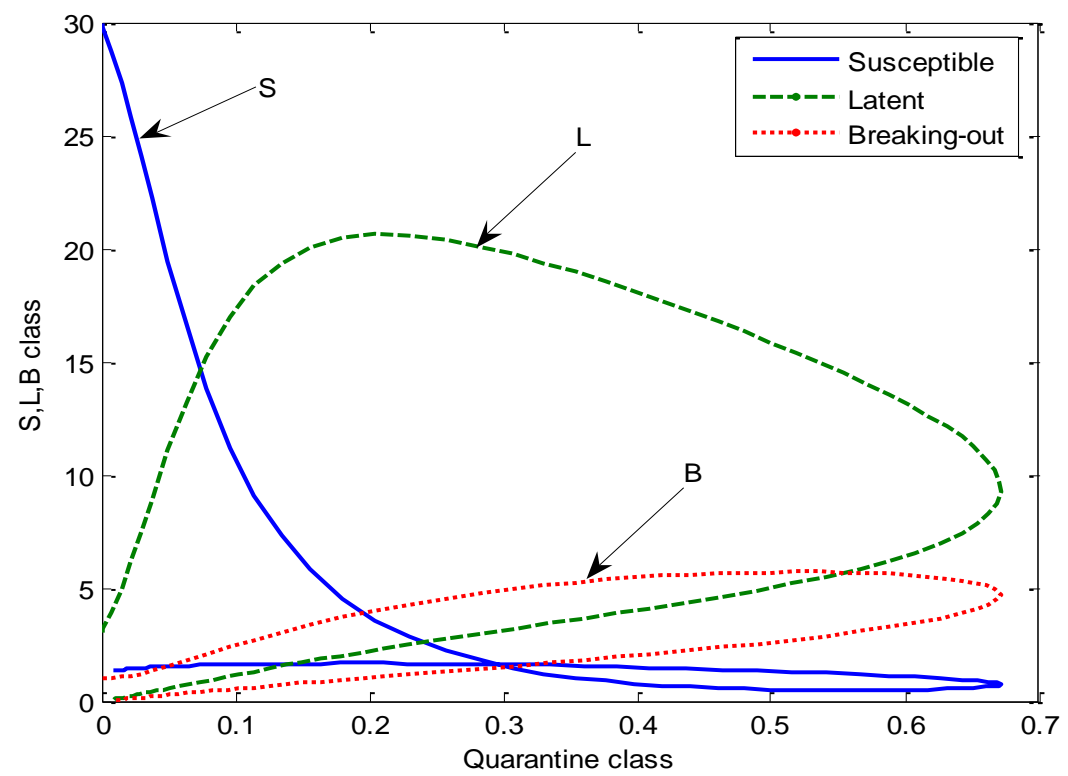

Figure 5. Effect of Quarantine Class on Different Classes When $\mu=0.1 ; B$ $=0.1 ; \mathrm{A}=0.3 ; \Gamma=0.1 ; \Lambda=0.2 ; \mathrm{H}=0.4 ; \Delta=0.4 ; \mathrm{E}=0.3$; 
Figure 5 Quarantine plays an important role in the recovery of the nodes. The quarantine nodes are treated with anti-malicious software and kept under constant observation.

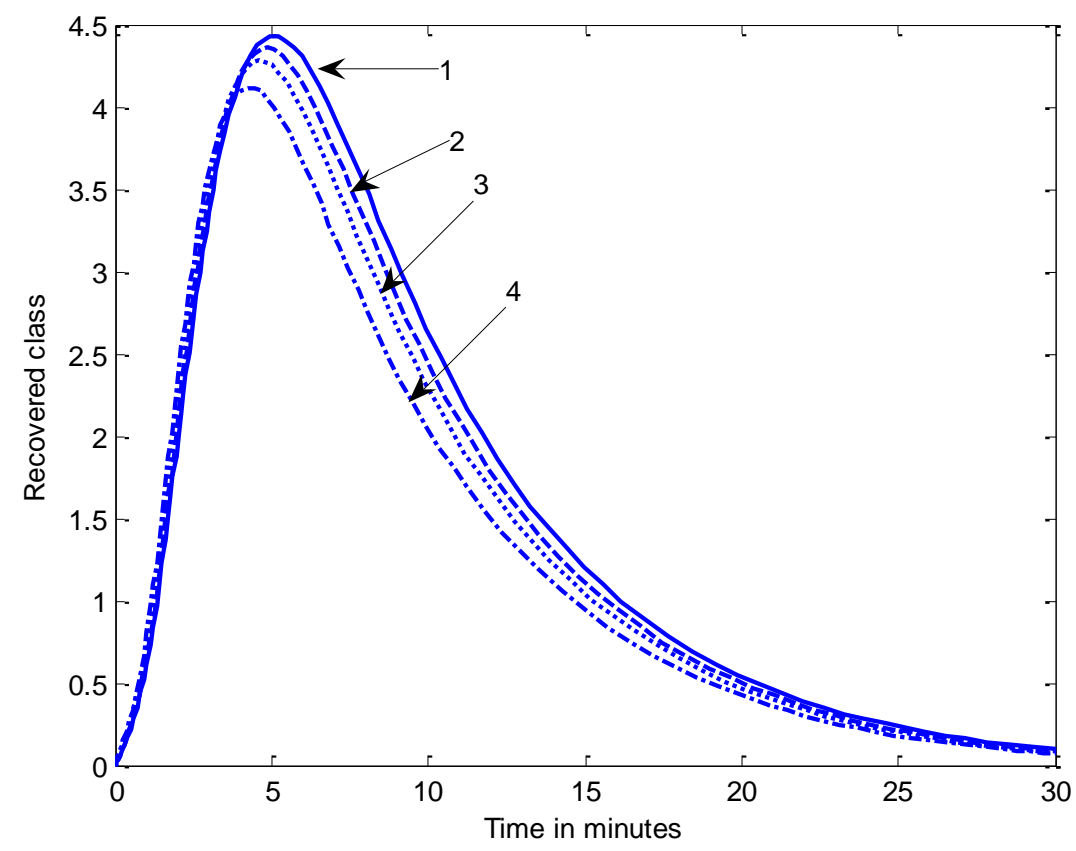

Figure 6. Dynamical Behavior of Recovered Class With Respect To Time

(1) $\mu=0.1 ; \beta=0.1 ; \alpha=0.3 ; \gamma=0.1 ; \lambda=0.2 ; \eta=0.4 ; \delta=0.4 ; \varepsilon=0.3$;

(2) $\mu=0.1 ; \beta=0.1 ; \alpha=0.3 ; \gamma=0.1 ; \lambda=0.2 ; \eta=0.45 ; \delta=0.4 ; \varepsilon=0.35$;

(3) $\mu=0.1 ; \beta=0.1 ; \alpha=0.3 ; \gamma=0.1 ; \lambda=0.2 ; \eta=0.5 ; \delta=0.4 ; \varepsilon=0.4$;

(4) $\mu=0.1 ; \beta=0.1 ; \alpha=0.3 ; \gamma=0.1 ; \lambda=0.2 ; \eta=0.6 ; \delta=0.4 ; \varepsilon=0.5$;

Figure 6 shows the analysis of recovered class. We observe the recovery rate is very high under different setting of parameter.

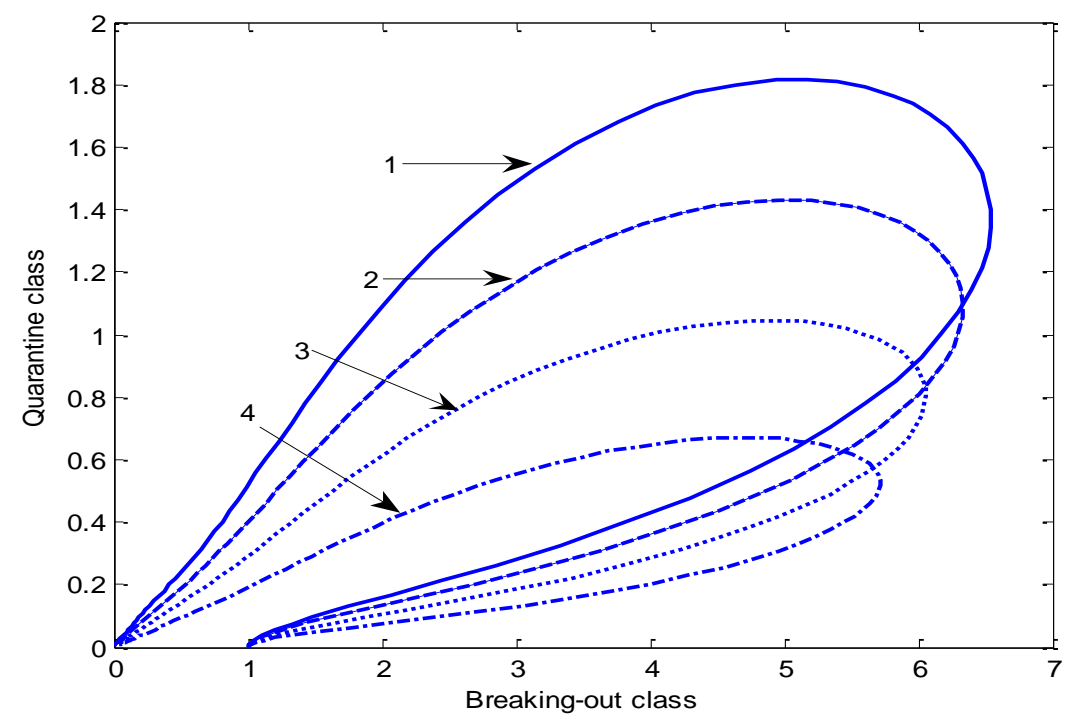

Figure 7. Dynamical Behavior of Breaking-Out Class versus Quarantine Class 
(1) $\mu=0.1 ; \beta=0.1 ; \alpha=0.3 ; \gamma=0.1 ; \lambda=0.2 ; \eta=0.4 ; \delta=0.4 ; \varepsilon=0.3$;

(2) $\mu=0.1 ; \beta=0.1 ; \alpha=0.35 ; \gamma=0.15 ; \lambda=0.2 ; \eta=0.45 ; \delta=0.4 ; \varepsilon=0.35$;

(3) $\mu=0.1 ; \beta=0.1 ; \alpha=0.4 ; \gamma=0.2 ; \lambda=0.2 ; \eta=0.5 ; \delta=0.4 ; \varepsilon=0.4$;

(4) $\mu=0.1 ; \beta=0.1 ; \alpha=0.45 ; \gamma=0.25 ; \lambda=0.2 ; \eta=0.6 ; \delta=0.4 ; \varepsilon=0.5$;

Figure 7 shows the relationship between breaking-out class and quarantine class under different setting of parameters and observe that infection in the quarantine class recovered highly after breaking-out period of virus.

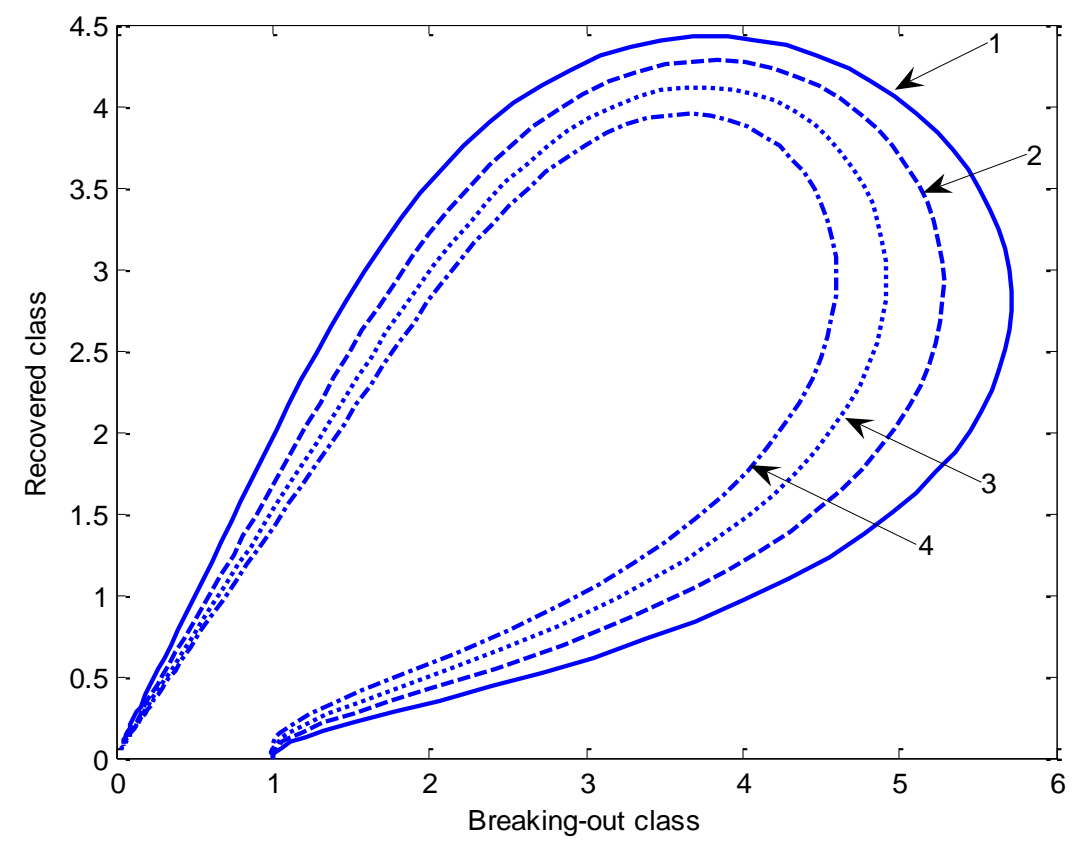

Figure 8. Dynamical Behavior of Breaking-Out Class versus Recovered Class

(1) $\mu=0.1 ; \beta=0.1 ; \alpha=0.3 ; \gamma=0.1 ; \lambda=0.2 ; \eta=0.4 ; \delta=0.4 ; \varepsilon=0.3$;

(2) $\mu=0.1 ; \beta=0.1 ; \alpha=0.3 ; \gamma=0.1 ; \lambda=0.2 ; \eta=0.5 ; \delta=0.4 ; \varepsilon=0.4$;

(3) $\mu=0.1 ; \beta=0.1 ; \alpha=0.3 ; \gamma=0.1 ; \lambda=0.2 ; \eta=0.6 ; \delta=0.4 ; \varepsilon=0.5$;

(4) $\mu=0.1 ; \beta=0.1 ; \alpha=0.3 ; \gamma=0.1 ; \lambda=0.2 ; \eta=0.7 ; \delta=0.4 ; \varepsilon=0.6$;

Figure 8 shows the relationship between breaking-out class and recovered class under different setting of parameters. We observe that recovery is very high after breaking-out period of virus. 


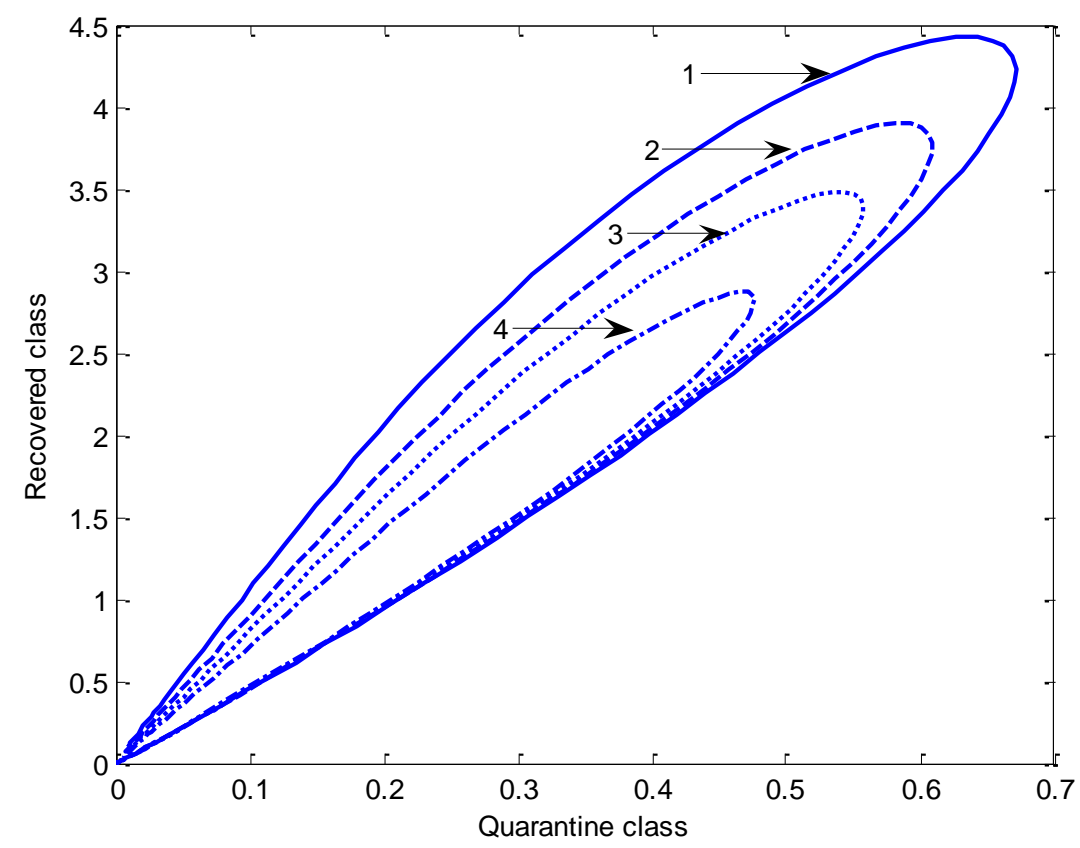

Figure 9. Dynamical Behavior of Quarantine Class versus Recovered Class

(1) $\mu=0.1 ; \beta=0.1 ; \alpha=0.3 ; \gamma=0.1 ; \lambda=0.2 ; \eta=0.4 ; \delta=0.4 ; \varepsilon=0.3$;

(2) $\mu=0.1 ; \beta=0.1 ; \alpha=0.3 ; \gamma=0.1 ; \lambda=0.2 ; \eta=0.4 ; \delta=0.5 ; \varepsilon=0.4$;

(3) $\mu=0.1 ; \beta=0.1 ; \alpha=0.3 ; \gamma=0.1 ; \lambda=0.2 ; \eta=0.4 ; \delta=0.6 ; \varepsilon=0.5$;

(4) $\mu=0.1 ; \beta=0.1 ; \alpha=0.3 ; \gamma=0.1 ; \lambda=0.2 ; \eta=0.4 ; \delta=0.8 ; \varepsilon=0.7$;

Figure 9 shows that quarantine plays an important role in the recovery of the nodes. By quarantine we achieve fast recovery. The more we quarantine the highly infectious nodes, the more is the recovery.

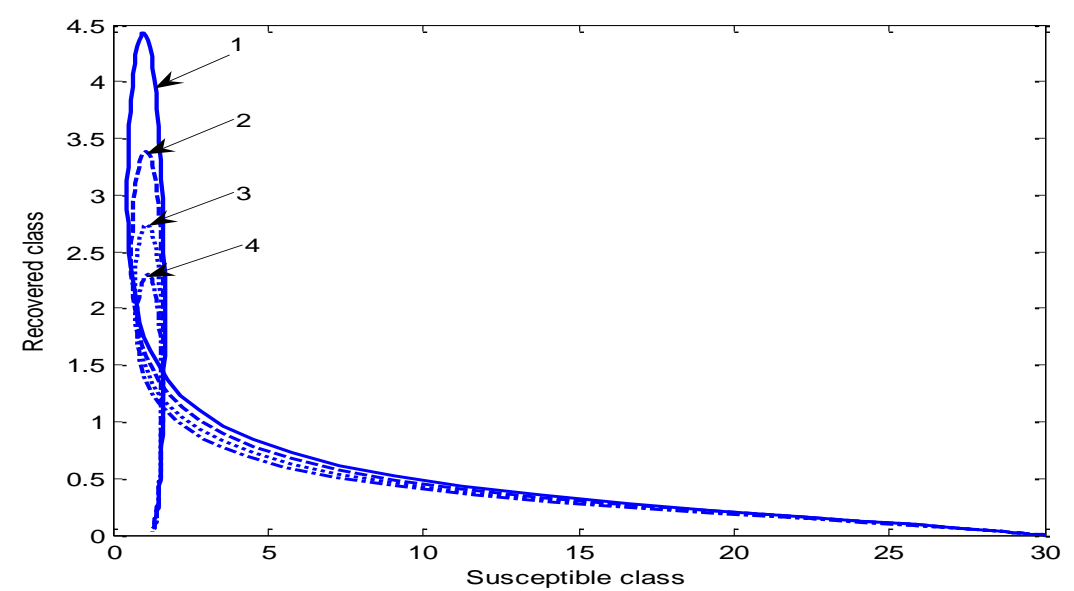

Figure 10. Dynamical Behavior of Susceptible Class versus Recovered Class

(1) $\mu=0.1 ; \beta=0.1 ; \alpha=0.3 ; \gamma=0.1 ; \lambda=0.2 ; \eta=0.4 ; \delta=0.4 ; \varepsilon=0.3$;

(2) $\mu=0.1 ; \beta=0.1 ; \alpha=0.3 ; \gamma=0.1 ; \lambda=0.2 ; \eta=0.4 ; \delta=0.4 ; \varepsilon=0.5$;

(3) $\mu=0.1 ; \beta=0.1 ; \alpha=0.3 ; \gamma=0.1 ; \lambda=0.2 ; \eta=0.4 ; \delta=0.4 ; \varepsilon=0.7$;

(4) $\mu=0.1 ; \beta=0.1 ; \alpha=0.3 ; \gamma=0.1 ; \lambda=0.2 ; \eta=0.4 ; \delta=0.4 ; \varepsilon=0.9$; 
Figure 10 shows that as the recovery rate increases the susceptibility towards infection decreases gradually to zero.

\section{Discussions}

As was indicated in the previous section, it is critical to take various actions to control the system parameters so that $R_{0}$ is remarkably below one. This section is intended to propose some effective measures for achieving this goal.

For our purpose, it is instructive to examine the sensitivities of $R_{0}$ to six system parameters: $\beta, \alpha, \gamma, \eta, \lambda$ and $\mu$, respectively. Following Arriola and Hyman [17], the normalized forward sensitivity indices with respect to $\beta, \alpha, \gamma, \eta, \lambda$ and $\mu$ are calculated, respectively, as follows:

$$
\begin{gathered}
\frac{\partial R_{0} / R_{0}}{\partial \beta / \beta}=\frac{\beta}{R_{0}} \frac{\partial R_{o}}{\partial \beta}=\frac{\beta}{R_{0}} \frac{\mu+\alpha+\gamma+\eta+\lambda}{(\mu+\alpha)(\mu+\gamma+\eta+\lambda)}=1>0, \\
\frac{\partial R_{0} / R_{0}}{\partial \alpha / \alpha}=\frac{\alpha}{R_{0}} \frac{\partial R_{0}}{\partial \alpha}=\frac{-\alpha(\gamma+\eta+\lambda)}{(\mu+\alpha)(\mu+\alpha+\gamma+\eta+\lambda)}<0, \\
\frac{\partial R_{0} / R_{0}}{\partial \gamma / \gamma}=\frac{\gamma}{R_{0}} \frac{\partial R_{0}}{\partial \gamma}=\frac{-\alpha \gamma}{(\mu+\gamma+\eta+\lambda)(\mu+\alpha+\gamma+\eta+\lambda)}<0, \\
\frac{\partial R_{0} / R_{0}}{\partial \eta / \eta}=\frac{\eta}{R_{0}} \frac{\partial R_{0}}{\partial \eta}=\frac{-\alpha \eta}{(\mu+\gamma+\eta+\lambda)(\mu+\alpha+\gamma+\eta+\lambda)}<0, \\
\frac{\partial R_{0} / R_{0}}{\partial \lambda / \lambda}=\frac{\lambda}{R_{0}} \frac{\partial R_{0}}{\partial \lambda}=\frac{-\alpha \lambda}{(\mu+\gamma+\eta+\lambda)(\mu+\alpha+\gamma+\eta+\lambda)}<0, \\
\frac{\partial R_{0} / R_{0}}{\partial \mu / \mu}=\frac{\mu}{R_{0}} \frac{\partial R_{0}}{\partial \mu}=\frac{\left.-\mu[\mu+\alpha)^{2}+(\gamma+\eta+\lambda)(2 \mu+\alpha+\gamma+\eta+\lambda)\right]}{(\mu+\alpha)(\mu+\gamma+\eta+\lambda)(\mu+\alpha+\gamma+\eta+\lambda)}<0,
\end{gathered}
$$

It can be seen that, among these four parameters, $R_{0}$, in proportion with $\beta$, is the most sensitive to the change in $\beta$. As opposed to this, the other five parameters $\alpha, \gamma, \eta$, $\lambda$ and $\mu$ have an inversely proportional relationship with $R_{0}$, an increase in $\alpha$ or $\gamma$ or $\eta$ or $\lambda$ or $\mu$ will bring about a decrease in $R_{0}$, with a proportionally smaller size of decrease.

Below, let us explain how these properties of model (2) can be utilized to control the spread of computer virus.

- Filtering and blocking suspicious messages with firewall located at the gateway of a domain, the parameter $\beta$ can be kept low and, hence, the chance that a virus-free computer within the domain is infected by a viral computer outside the domain can be significantly decreased, yielding a lower threshold value $R_{0}$.

- Timely updating and running antivirus software of the newest version on computers, the breaking out rate of latent computers, $\alpha$, and the cure rate of breaking out computers, $\gamma$, can be remarkably enhanced, leading to a low $R_{0}$.

- Timely disconnecting computers from the Internet when the connections are unnecessary, the disconnecting rate of computers, $\mu$, can be made high, bringing about an ideal $R_{0}$. 
In practice, all of these measures are strongly recommended to achieve a threshold value well below unity, so that viruses within the Internet approach extinction.

\section{Conclusion}

A dynamical Epidemic Computer Virus model, the SLBQRS model for the transmission of viruses over the internet is formulated. In nearly all previous computer virus propagation models with latent compartment, to our knowledge, latent computers are assumed not to infect other computers, which does not accord with the real situations. To overcome this defect, the SLBQRS model proposed in this paper assumes that all latent computers have infectivity. The model addresses the worm containment based on the dynamic quarantine on the node in a group that has exhibited highly infectious behavior. By Quarantine we achieve fast recovery and end the susceptibility of spread of infection to benign nodes. The dynamics of this model has been fully studied. The results concerning this model include the following. (1) Two equilibria, the virus-free equilibrium $\mathrm{E}_{0}$ and the viral equilibrium $\mathrm{E}^{*}$, as well as the basic reproduction ratio $R_{0}$ are obtained. (2) The dynamical behavior is determined completely by the value of $R_{0}: R_{0} \leq 1$ implies the global stability of $\mathrm{E}_{0}$, whereas $R_{0}>1$ implies the global stability of $\mathrm{E}^{*}$. (3) By conducting a sensitive analysis of $R_{0}$ with respect to various model parameters and on the condition that $R_{0} \ll<1$, a series of measures of strategies is proposed for controlling the spread of virus through the Internet effectively.

\section{References}

[1] J. O. Kephart, S. R. White, and D. M. Chess, "Computers and epidemiology," IEEE Spectrum, pp. 20-26, (1993).

[2] X. Han and Q. Tan, "Dynamical behavior of computer virus on Internet," Applied Mathematics and Computation, vol. 217, no. 6, pp. 2520-2526, (2010).

[3] B. K. Mishra and N. Jha, "Fixed period of temporary immunity after run of anti-malicious software on computer nodes," Applied Mathematics and Computation, vol. 190, pp. 1207-1212, (2007).

[4] B. K. Mishra and S. K. Pandey, "Fuzzy epidemic model for the transmission of worms in computer network," Nonlinear Analysis, vol. 11, pp. 4335-4341, (2010).

[5] J. Ren, X. Yang, L.-X. Yang, Y. Xu, and F. Yang, "A delayed computer virus propagation model and its dynamics," Chaos, Solitons \& Fractals, vol. 45, no. 1, pp. 74-79, (2012).

[6] J. Ren, X. Yang, Q. Zhu, L.-X. Yang, and C. Zhang, "A novel computer virus model and its dynamics,"Nonlinear Analysis, vol. 13, no. 1, pp. 376-384, (2012).

[7] B. K. Mishra and S. K. Pandey, "Dynamic model of worms with vertical transmission in computer network," Applied Mathematics and Computation, vol. 217, no. 21, pp. 8438-8446,( 2011).

[8] B. K. Mishra and D. K. Saini, "SEIRS epidemic model with delay for transmission of malicious objects in computer network," Applied Mathematics and Computation, vol. 188, no. 2, pp. 14761482, (2007).

[9] F. Wang, Y. Zhang, C. Wang, J. Ma, and S. Moon, "Stability analysis of a SEIQV epidemic model for rapid spreading worms," Computers and Security, vol. 29, no. 4, pp. 410-418, (2010).

[10] B. K. Mishra and N. Jha, "SEIQRS model for the transmission of malicious objects in computer network," Applied Mathematical Modelling, vol. 34, no. 3, pp. 710-715,( 2010).

[11] L.-X. Yang, X. Yang, L. Wen, and J. Liu, "A novel computer virus propagation model and its dynamics,'International Journal of Computer Mathematics. In press.

[12] L.-X. Yang, X. Yang, L. Wen, and J. Liu, "Propagation behavior of virus codes in the situation that infected computers are connected to the Internet with positive probability," Discrete Dynamics in Nature and Society, vol. 2012, Article ID 693695, 13 pages, (2012).

[13] L.-X. Yang, X. Yang, Q. Zhu, and L. Wen, "A computer virus model with graded cure rates," Nonlinear Analysis: Real World Applications, vol. 14, no. 1, pp. 414-422, (2013).

[14] X. Yang and L.-X. Yang, "Towards the epidemiological modeling of computer viruses," Discrete Dynamics in Nature and Society, vol. 2012, Article ID 259671, 11 pages, (2012).

[15] M. Yang, Z. Zhang, Q. Li, G. Zhang, "An SLBRS Model with Vertical Transmission of Computer Virus over the Internet," Discrete Dyn. Nat. Soc. Vol. 2012, Article ID 925648, 17 pages,(2012).

[16] R.S. Varga, Matrix iterative analysis, Englewood cliffs, N.J: Prentice-Hall Inc., (1962)

[17] L. Arriola and J. Hyman, "Forward and adjoint sensitivity analysis with applications in dynamical systems," Lecture Notes in Linear Algebra and Optimization, (2005). 


\section{Authors}
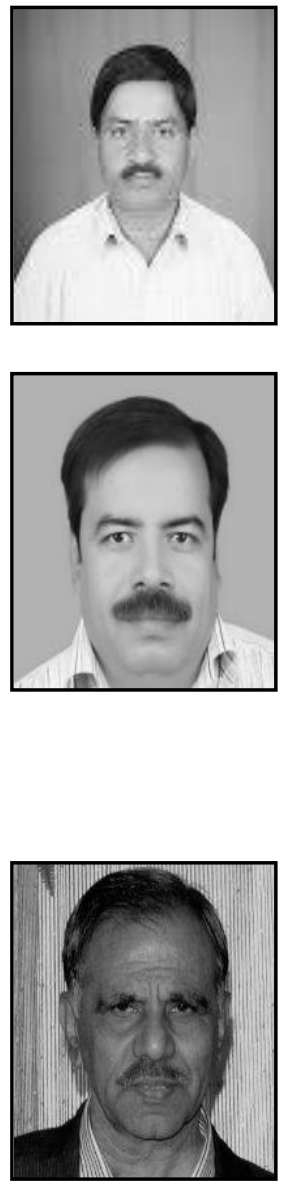

Munna Kumar is a research scholar pursuing his Ph. D. degree. He received his Master's in Mathematics from Vinoba Bhave University, Hazaribag, India. His research interests include Mathematical Modeling on cyber attack and defense.

Bimal Kumar Mishra is a Professor in the Department of Mathematics, Birla Institute of Technology, Mesra, Ranchi, India. $\mathrm{He}$ received his Master's in Mathematics and Master's in Operational Research from University of Delhi, India and earned his Ph. D. from Vinoba Bhave University, Hazaribag, India in 1997. He was awarded D. Sc. In 2007, from Berhampur University, India. $\mathrm{He}$ has published more than 100 research papers in journalsof high repute and conference proceedings. His research interests include Nonlinear Analysis and Bifurcation and presently working in the area of Cyber attack and defense.

T. C. Panda is a Retired Professor in the Department of Mathematics, Berhampur University, India. He is the President of Orissa Mathematical Society, Bhubaneshwar Area, India. He has published in journals of high repute and supervised several $\mathrm{Ph} . \mathrm{D}$. scholars. His research areas include Fluid Mechanics, and Nonlinear Analysis. 